\title{
Influence of Environmental and Electrochemical Factors in the Durability of Concrete
}

\author{
F.J. Olguín Coca, F. Almeraya-Calderón, V.M. Orozco Carmona, A. \\ Borunda, C. Gaona Tiburcio, A. Martínez Villafañe, A. Torres ${ }^{a}$ \\ División de Deterioro de Materiales e Integridad Estructural/Grupo Corrosión, Centro de \\ Investigación en Materiales Avanzados, S.C., (CIMAV), Miguel Cervantes 120, Complejo \\ Industrial Chihuahua 31109, Chihuahua, México \\ ${ }^{a}$ Instituto Mexicano del Transporte (IMT)
}

Received 26 February 2004; accepted in revised form 9 July 2004

\begin{abstract}
This work is part of the DURACON project, in which it is characterized the concretes exposed durability in the present iberoamerican environmental conditions, being based in the exhibition of armed specimens in at least two different atmospheres, a marine and an urban, in each iberoamerican participant country. The results obtained by exposing these armed concrete specimens to the environmental conditions of the city Chihuahua, Mexico, during the first 15 months of exhibition, will be discussed in this work. Two concretes were designed, one with a relationship w/c (water/cement) $=0.65$, and the other one with $\mathrm{w} / \mathrm{c}=0.45$, and three cover thickness $(1.5,2.0$ and $3.0 \mathrm{~cm})$. Tests were mainly based in measuring electrochemical parameters that allowed evaluating the corrosion kinetics of the bars, by means of potential corrosion measurements and implementing the technique of linear resistance polarization in order to determine the corrosion rate. All this information will permit, with the use of specific models, to design durable concrete structures, and to repair appropriately those already existent, what will rebound in a decrease of the economic losses that at the moment is causing the phenomenon of corrosion in our country.
\end{abstract}

Keywords: corrosion, durability, armed concrete.

\section{Introducción}

La corrosión de la armadura es una de las causas más habituales responsable del deterioro de las estructuras de hormigón que está llevando a elevados gastos de reparación y mantenimiento, con la preocupación que ello supone para las 
administraciones que son las propietarias de las obras públicas, y los particulares cuyas viviendas sufren deterioros prematuros.

Las investigaciones relacionadas con la corrosión de armaduras han sido amplias en los últimos diez años, pero han estado especialmente enfocadas 1) al estudio de las causas y mecanismos de deterioro, 2) al desarrollo de técnicas electroquímicas de evaluación y control (tanto en laboratorio como "in situ"), y 3) al empleo de métodos de protección. Más recientemente se planteó la necesidad de cuantificar la vida útil de estructuras afectadas por corrosión, por lo que en los últimos años se ha ido centrando el interés en la búsqueda de modelos que permitan predecir la vida útil de estructuras en servicio no deterioradas o estimar la futura en el caso de nuevas, influyendo desde la fase de proyecto.

Las características físicas del concreto como aislante están relacionadas con la estructura de la red de poros, que se genera debido al exceso de agua que es necesario utilizar en el amasado para hacer suficientemente trabajable el material. La porosidad total del concreto está constituida, tanto por los poros cerrados u ocluidos como por una red de poros, cuyos tamaños oscilan entre pocas $\mu \mathrm{m}$ hasta alrededor de $100 \mu \mathrm{m}$ (poros de gel) desde $\sim 100 \mu \mathrm{m}$ hasta $\sim 10^{5}$ $\mu \mathrm{m}$ (poros capilares), y desde $10^{5} \mu \mathrm{m}$ hasta el orden de $\mathrm{mm}$, que son los llamados poros de aire o grandes poros. Todos los que están comunicados entre sí están relacionados con el concepto de permeabilidad o penetrabilidad, que es la característica fundamental que va a controlar la llegada de agentes agresivos hasta la varilla. La permeabilidad del concreto a una determinada sustancia no depende solo del tamaño y número de poros, como en el caso de los materiales porosos inertes, sino que influyen en ella también fenómenos de absorción, las reacciones químicas que se pueden producir, el necesario equilibrio de capas eléctricas, etc. Todo ello hace que algunos autores [1-3] consideren que el concreto se comporta como una membrana semipermeable.

Cuando la humedad ambiental es suficiente, estos poros del hormigón están rellenos por un líquido acuoso constituido principalmente por iones $\mathrm{OH}^{-}$ (alcalinidad elevada con un $\mathrm{pH}$ entre 12 y 14 ), $\mathrm{Ca}^{2+}, \mathrm{Na}^{+}, \mathrm{K}^{+}$y $\mathrm{SO}_{4}{ }^{2-}$. $\mathrm{La}$ cantidad relativa de cada uno de ellos depende fundamentalmente del tipo y 
proporción de cemento utilizado y de la relación agua/cemento, además, esta composición varía con la edad del hormigón, habiéndose demostrado que a partir de los 28 días de edad, el ión $\mathrm{Ca}^{2+}$ prácticamente desaparece de la disolución, que pasa a estar constituida casi completamente por $\mathrm{NaOH}$ y $\mathrm{KOH}$. A esta elevada alcalinidad y con el contenido de $\mathrm{O}_{2}$ normal en las estructuras enterradas o sumergidas, el acero se recubre de una capa de óxidos muy adherente, compacta e invisible (pasivante) que lo preserva indefinidamente de cualquier signo de corrosión, mientras el hormigón sea de buena calidad, no esté fisurado y no cambie sus características físicas o químicas por agresiones exteriores [4].

Cuando las condiciones de servicio cambian y el concreto se altera, o a través de él penetran sustancias agresivas, la corrosión de las varillas se desencadena con una triple consecuencia: a) el acero disminuye su sección o incluso se convierte completamente en óxido; b) debido a las presiones que ejerce el óxido al generarse, el concreto puede fisurarse o desintegrarse; y c) la adherencia varillahormigón disminuye o desaparece. Por otra parte, la carbonatación también es un problema que actualmente está afectando a muchas estructuras en nuestros países, por esa diversidad de climas que poseemos, aunque en menor escala que el problema con los cloruros, pero se tienen dificultades similares para su predicción de la carbonatación. Así, estos problemas tienen una relación directa con los parámetros metereoquímicos del ambiente al cual se encuentran expuestas las estructuras.

Se han dado distintas definiciones al concepto de vida en servicio o vida útil relacionada con la idea de durabilidad de una estructura de hormigón [5]. Desde el punto de vista de la corrosión de las varillas se ha propuesto un modelo simple, se está intentando definir todos los parámetros que llevarían a poder calcular previamente la vida en servicio o la durabilidad de una determinada estructura en condiciones de servicio con un coeficiente de seguridad suficiente. Esto en conjunto con los modelos que se definirán en el proyecto y los resultados de la evaluación físico-química y electroquímica de las diferentes probetas expuestas, permitirán definir una mejor forma de diseñar obras en 
concreto durables y reparar adecuadamente las ya existentes que presentan este problema.

En la actualidad es de vital importancia para obtener la información faltante en cuanto al deterioro producido en las infraestructuras de concreto en general y su interdependencia con el ambiente en el que están expuestas. El hecho de poder contar con manuales que consideren el efecto del medio ambiente como parámetro de diseño estructural ha sido poco estudiado en todo el mundo, no solo en países en vías de desarrollo como México. Este proyecto sería la punta de lanza que nos ayudaría a estar en la vanguardia en este rubro de durabilidad de hormigón que, en el ámbito mundial, ha estado desarrollándose de una manera continua.

Cabe aclarar que este proyecto tiene carácter internacional, ya que en la actualidad el CYTED aprobó el Proyecto XV.3 Influencia de la Acción del Medio Ambiente en la Durabilidad del Hormigón, también llamado DURACON, en donde el IMT (Instituto Mexicano del Trasporte) está fungiendo como coordinador de la parte Mexicana y CIMAV participa con una estación de monitoreo urbano-industrial, la cual cubrirá el Norte del País, considerando el clima tan extremoso que se tiene en esta región. Posteriormente se realizara un comparativo con las demás estaciones de México.

\section{Materiales y métodos}

La estación de ensayo natural fue constituida por paneles metálicos para sostener los especimenes de concreto a un metro por encima del suelo, y orientados hacia la dirección de los vientos predominantes. La estación de CIMAV se encuentra ubicada en el Complejo Industrial Chihuahua (atmósfera urbano-industrial).

\section{Materiales para la fabricación de especimenes de concreto}

Los concretos estudiados fueron fabricados tomando en cuenta las especificaciones que se dan a continuación: 1) Tipo de Cemento: Portland I (Superficie específica Blaine a 3600 y 8\% de $\mathrm{C}_{3} \mathrm{~A}$ ); 2) Árido siliceo triturado de 19 mm (grueso); 3) Arena cuarzosa; 4) Relación a/c: 0.45 y 0.65; 5) Adición de 
plastificante $0.3 \%$ vs. cemento (tipo Plastiment VZ de Sika o similar); 6) Asentamiento 10 más o menos $1.0 \mathrm{~cm}$; 7) Encofrado en madera resinosa; 8) Curado bajo agua por 7 días; 9) Se prepararon 6 vigas de concreto con armadura (tres con relación a/c $=0.45$ y tres con relación a/c $=0.65$ ).

\section{Especimenes de hormigón para las estaciones de ensayo natural}

Los especimenes de hormigón que fueron expuestos en las estaciones de ensayo natural son: especimenes prismáticos de $15 \times 15 \times 30 \mathrm{~cm}$ con 3 armaduras, con espesores de recubrimiento de 1.5, 2 y $3 \mathrm{~cm}$ (ver Fig. 1 y 2 ).

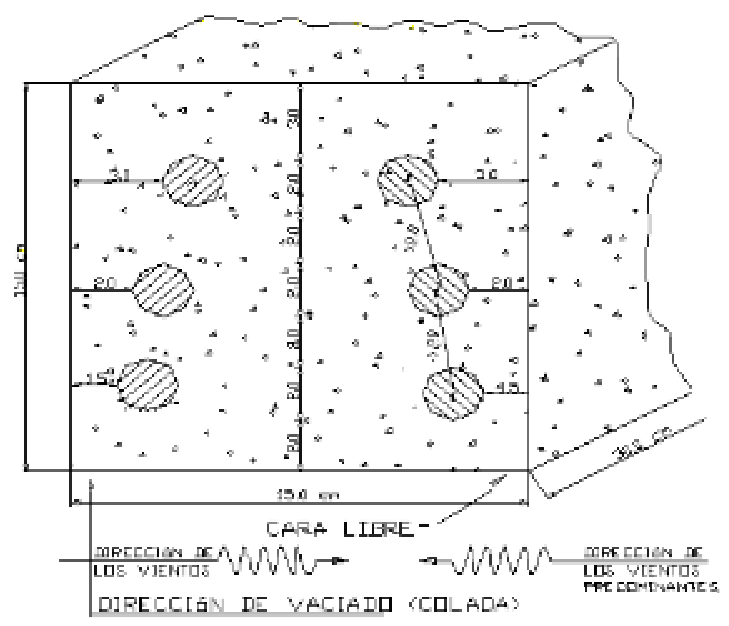

Figura 1. Sección transversal de la viga.

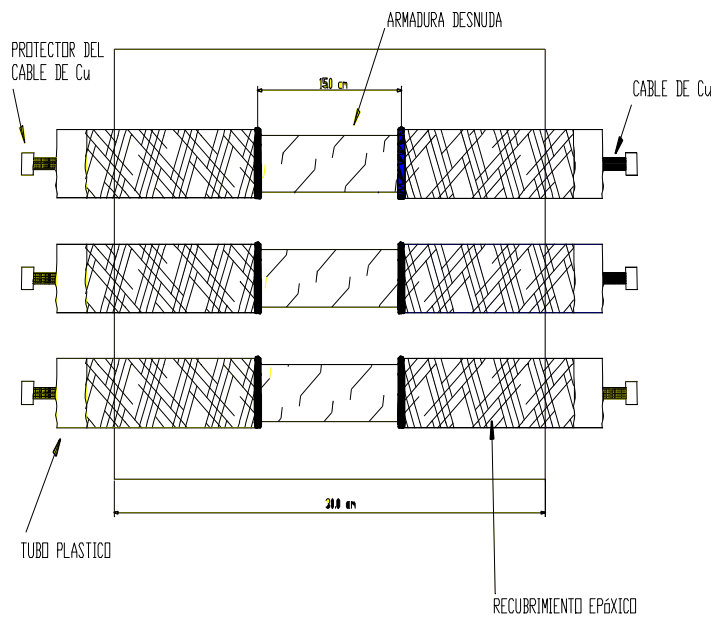

Figura 2. Sección longitudinal de la viga. 


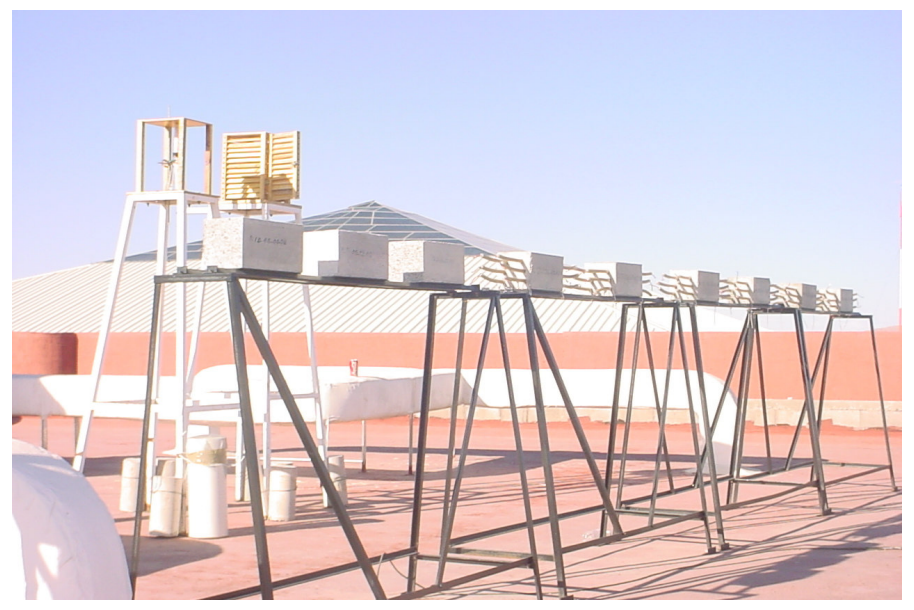

Figura 3. Estación de monitoreo CIMAV (atmósfera urbano-industrial).

Para cada composición de hormigón objeto de estudio, se fabricaron 3 especimenes con armadura y diferente relación agua/cemento y se expusieron 6 especimenes de hormigón (ver Fig. 3).

Ensayos a realizar sobre los especimenes de concreto expuestos en las estaciones de ensayo natural

Los especimenes de hormigón con armaduras se expondrán en las estaciones de ensayo natural por un período de hasta cuatro años. Durante este período, se realizarán ensayos para determinar ciertos parámetros de durabilidad, como se especifica a continuación:

1. potencial de media celda (ASTM C 876-91) [6];

2. potencial de media celda y resistencia de polarización lineal (GECOR).

\section{Resultados y discusion}

\section{Potencial de media celda (potenciales de corrosión)}

El análisis de las gráficas de potencial obtenidas de cada una de las vigas en estudio, se realizó de acuerdo a los criterios de evaluación establecidos por la norma ASTM C-876-91 [6], ver tabla 1.

En la gráfica 1 (inciso a-c) se observa el comportamiento del potencial a lo largo de un año de exposición para las vigas 1,2 y 3 con la relación a/c 0.45 y con los tres recubrimientos $(1.5,2.0$ y $3.0 \mathrm{~cm})$. Los valores de los potenciales no superaron los $-200 \mathrm{mV}$ lo que indica que existe un $10 \%$ de probabilidad de que 
exista corrosión de acuerdo con ASTM C876-91. Los potenciales en las vigas ya mencionadas comenzaron con valores de aproximadamente $-140 \mathrm{mV}$ al inicio del estudio, llegando a alcanzar valores de $120 \mathrm{mV}$ después de 15 meses de exposición; esto significa que el acero refuerzo se pasivo (ver gráfica 1).

Tabla 1. Interpretación de resultados norma ASTM C876-91.

\begin{tabular}{cc}
\hline $\begin{array}{c}\text { Potencial de media celda } \\
(\mathrm{mV})\end{array}$ & Riesgo de daño \\
\hline$<-200$ & $10 \%$ de probabilidad de corrosión \\
-200 a -350 & cierta incertidumbre \\
$>-350$ & $90 \%$ de probabilidad de corrosión \\
\hline
\end{tabular}

En las vigas con relación a/c de 0.65 (gráfica 2, incisos a-c) se observa que los valores son más negativos que en la relación a/c de 0.45 . Los valores de los potenciales permiten observar que existe un $10 \%$ de probabilidad de corrosión de acuerdo como se establece en ASTM C876-91. El comportamiento de los potenciales, que se observó en estas tres vigas (4-6), fue de una tendencia a la formación de una película pasiva sobre la superficie de las varillas de refuerzo y, de acuerdo con las condiciones climatológicas de la región, el porcentaje de humedad relativa $(\mathrm{HR})$ disminuye drásticamente lo que ocasiona que el sistema de corrosión sea más lento.

\section{Velocidad de Corrosión}

El análisis de las graficas de $\mathrm{i}_{\text {corr }}$ obtenidas de cada una de las vigas en estudio, se realizo de acuerdo a la referencia internacional DURAR, ver tabla 2 [7].

Tabla 2. Clasificación de $\mathrm{i}_{\text {corr }}$ en términos de vida útil (DURAR).

\begin{tabular}{cc}
\hline $\mathrm{i}_{\text {corr }}\left(\mu \mathrm{A} / \mathrm{cm}^{2}\right)$ & Nivel de corrosión \\
\hline$<0.1$ & Despreciable \\
$0.1-0.5$ & Moderado \\
$0.5-1$ & Elevada \\
$>1$ & Muy elevada \\
\hline
\end{tabular}




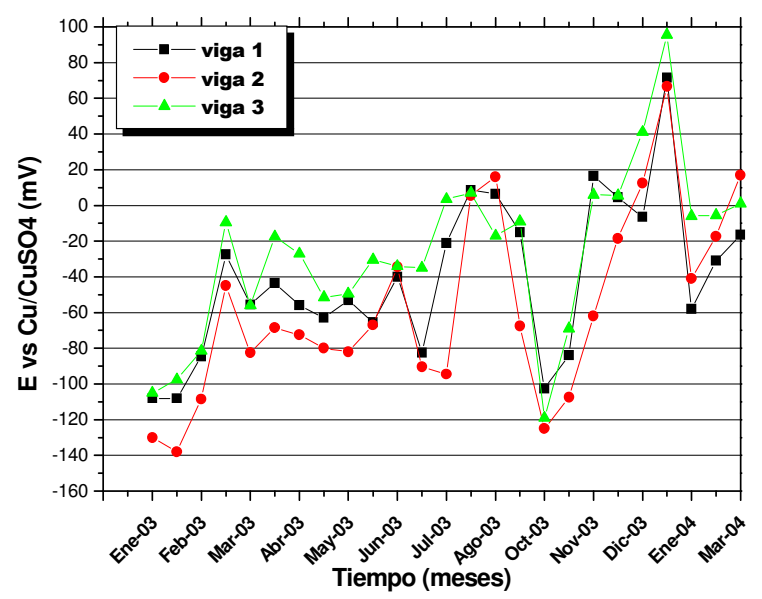

(a). Recubrimiento $1.5 \mathrm{~cm}$

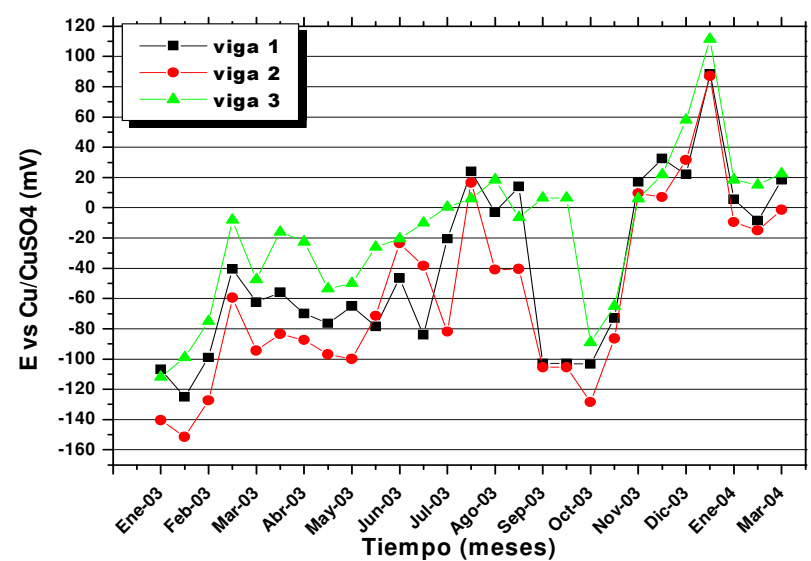

(b). Recubrimiento $2.0 \mathrm{~cm}$

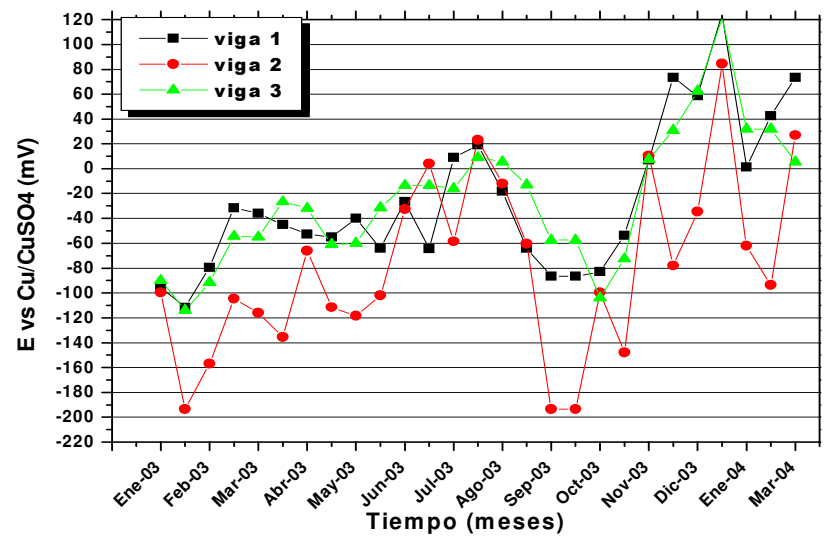

(c). Recubrimiento $3.0 \mathrm{~cm}$

Gráfica 1. Comportamiento del potencial de corrosión en las vigas 1 - 3. Rel. a/c 0.45. 


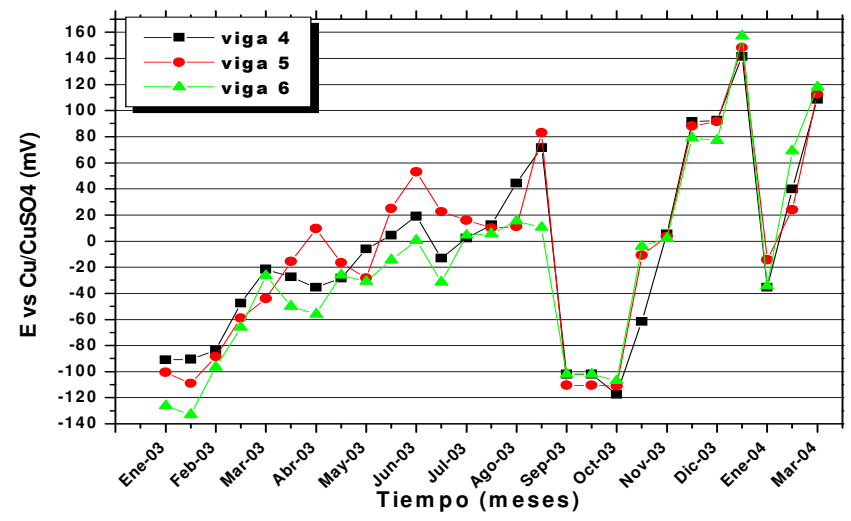

(a). Recubrimiento $1.5 \mathrm{~cm}$

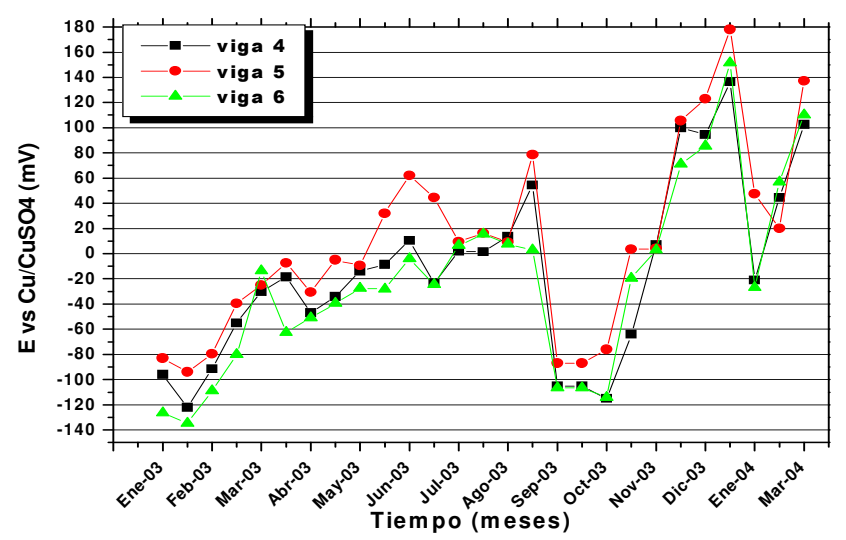

(b). Recubrimiento $2.0 \mathrm{~cm}$

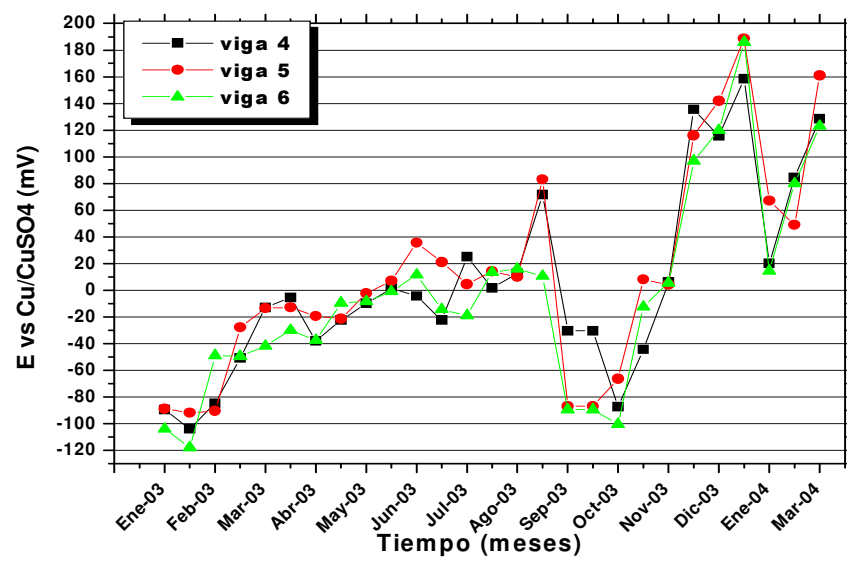

c). Recubrimiento $3.0 \mathrm{~cm}$

Gráfica 2. Comportamiento del potencial de corrosión en las vigas 4 - 6. Rel. a/c 0.65. 
En la gráfica 3 (incisos a-c) correspondientes a las vigas 1-3 con Rel. a/c de 0.45 se observan los siguientes comportamientos de $\mathrm{i}_{\text {corr }}$. En el Rec. de $1.5 \mathrm{~cm}$ (inciso a), la $i_{\text {corr }}$ se encuentra en el intervalo de 0.10 a $0.45 \mu \mathrm{A} / \mathrm{cm}^{2}$, lo cual permite clasificarlo como una corrosión moderada, basándose en los criterios del DURAR. El Rec. de $2.0 \mathrm{~cm}$ tuvo un comportamiento descendente, lo que permite identificar que la corrosión de la varilla se esta pasivando (corrosión moderada) dado que la $i_{\text {corr }}$ esta en un intervalo de 0.1 a $0.5 \mu \mathrm{A} / \mathrm{cm}^{2}$, y el Rec. de $3.0 \mathrm{~cm}$, inciso c, comienza con una velocidad de corrosión elevada $\left(0.5 \mu \mathrm{A} / \mathrm{cm}^{2}\right)$ los primeros 4 meses de exposición, y con el paso del tiempo se vuelve una corrosión moderada. En el caso de las otras 2 vigas el comportamiento se muestra de manera muy diferente debido a que sus valores no varían con respecto al tiempo presentando una velocidad de corrosión de tipo moderada.

En las vigas 4-6 con Rel. a/c de 0.65 (ver gráfica 4, incisos a-c) el comportamiento del recubrimiento de $1.5 \mathrm{~m}$ para las vigas 4-6 se observa que los valores de velocidad de corrosión son de manera moderada, pero entre los meses 8-10 (Agosto-Octubre) esa velocidad de corrosión se acelero sin llegar a una velocidad de corrosión elevada y en los siguientes meses volvió a una pasivacion dando por consiguiente una velocidad moderada. En las vigas 4-6 con Rec. de $2.0 \mathrm{~cm}$ (inciso b), presentaron las siguientes características: la viga 5 tuvo un comportamiento más activo que en las otras 2 vigas siendo que los valores de $\mathrm{i}_{\text {corr }}$ son más altos en la viga 2 . Pero la velocidad de corrosión fue moderada para las 3 vigas exceptuando los meses de Abril-Agosto donde la velocidad fue despreciable según lo establece el DURAR. En el inciso c, de la gráfica 4, el Rec. de $3.0 \mathrm{~cm}$ tuvo un comportamiento similar al del Rec. 2.0 en la velocidad de corrosión moderada. 


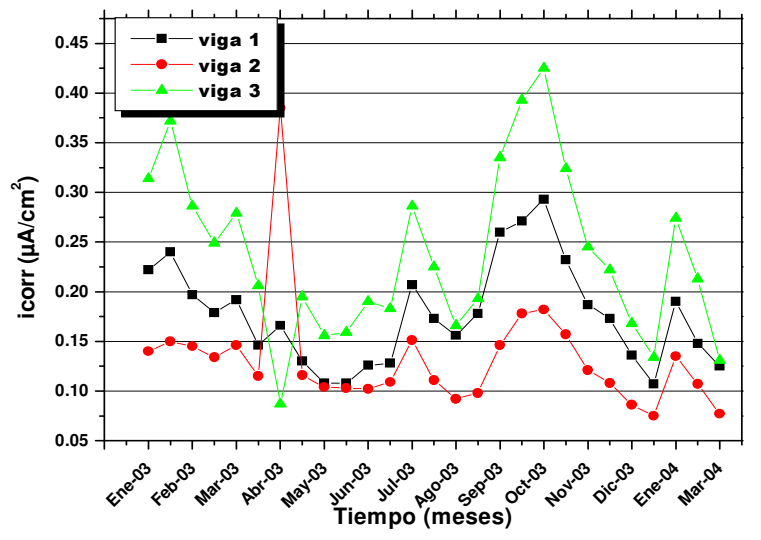

(a). Recubrimiento $1.5 \mathrm{~cm}$

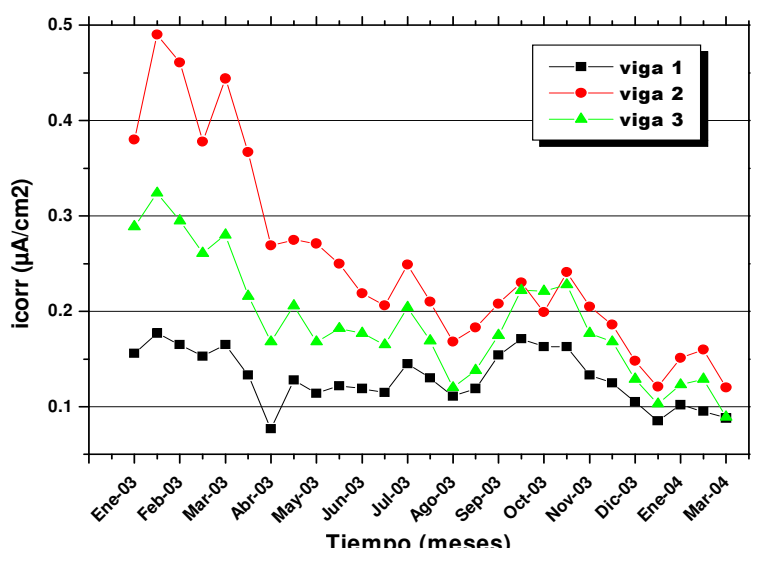

(b). Recubrimiento $2.0 \mathrm{~cm}$

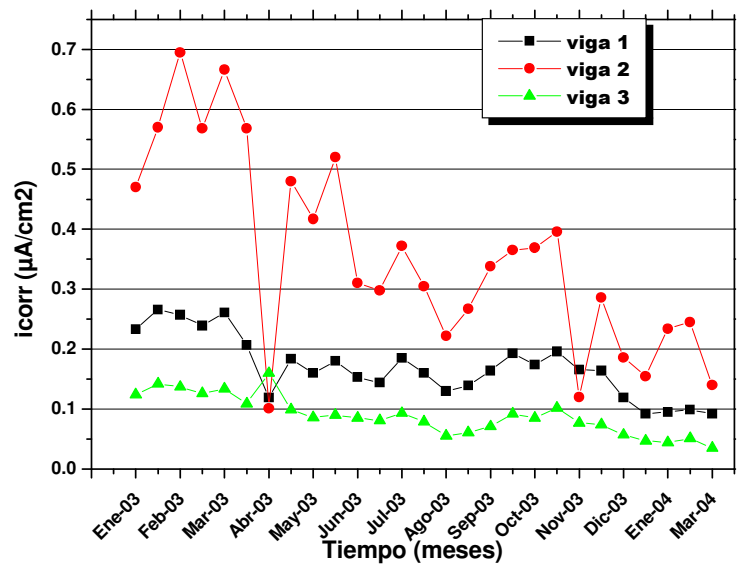

c). Recubrimiento $3.0 \mathrm{~cm}$

Gráfica 3. Comportamiento de la intensidad de corriente $i_{\text {corr }}$ en las vigas 1-3. Rel. a/c 0.45 . 


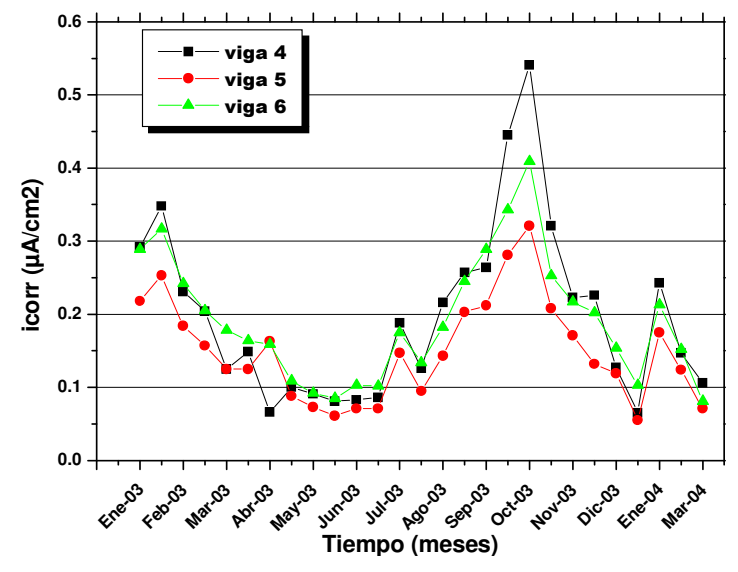

(a). Recubrimiento $1.5 \mathrm{~cm}$

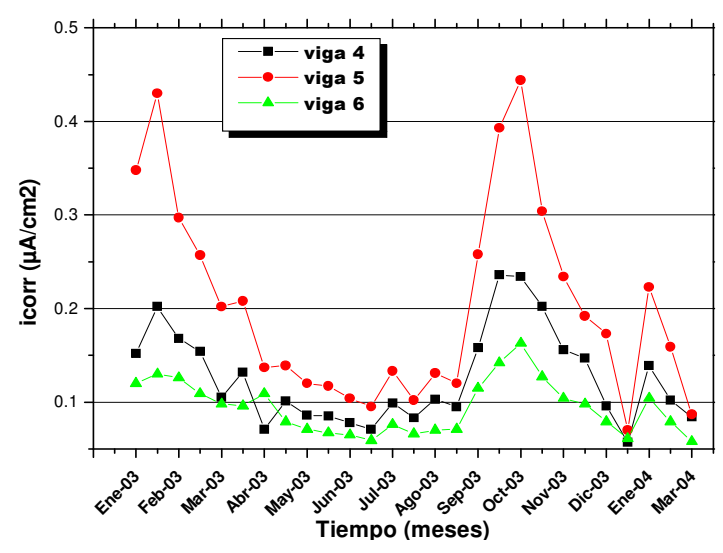

(b). Recubrimiento $2.0 \mathrm{~cm}$

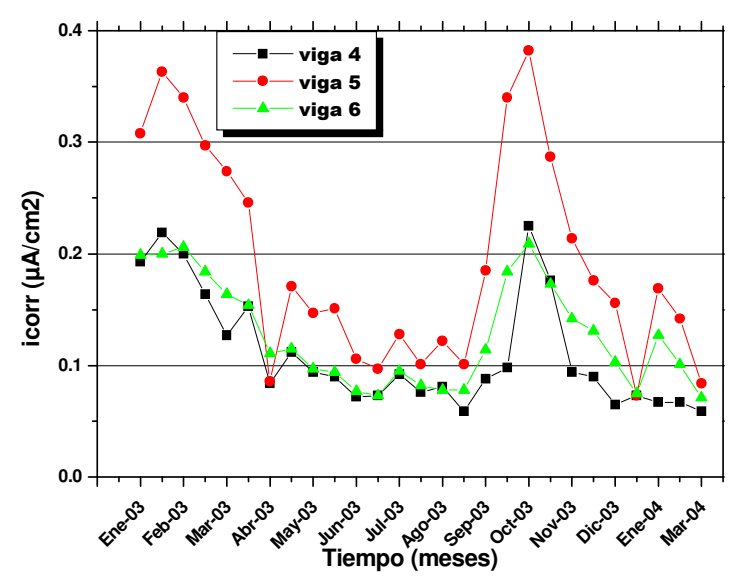

(c). Recubrimiento $3.0 \mathrm{~cm}$

Gráfica 4. Comportamiento de la intensidad de corriente $i_{\text {corr }}$ en las vigas 4-6. Rel. a/c 0.65 . 


\section{Conclusiones}

Los valores de los potenciales de corrosión observados en las varillas de refuerzo de las vigas con una relación a/c $=0.65$, se comportan más activos en los primeros días de exposición. Sin embargo conforme aumenta el tiempo de exposición los potenciales se vuelven más nobles, tendiendo a presentar un 10\% de probabilidad corrosión, de acuerdo con los criterios de interpretación ASTM C876-91, siendo el mismo caso para las vigas con una relación $\mathrm{a} / \mathrm{c}=0.45$. Hasta la última fecha de monitoreo mostrada en esta investigación, no se observa ninguna influencia del recubrimiento en el comportamiento de los valores del potencial.

La intensidad de corrosión para las seis vigas con las dos relaciones a/c y los tres tipos de recubrimientos, mostraron velocidades de corrosión moderadas, según a los criterios establecidos por la referencia internacional de valores de corrosión

DURAR. De igual manera, los diversos recubrimientos no influyen en los valores de la $\mathrm{i}_{\text {corr }}$.

Humedad relativa (HR) es alta, los potenciales de corrosión en las seis vigas y con los tres recubrimientos tienden a ser más activos, y cuando la humedad relativa (HR) es baja estos potenciales son más nobles. Y en contraparte, se observó que a medida que la humedad relativa disminuye la intensidad de corrosión es baja y cuando la humedad es alta la intensidad de corrosión se incrementa, por lo cual el sistema de corrosión acero-concreto se vuelve activo.

\section{Agradecimientos}

Los autores agradecen el apoyo económico al CIMAV, CONACYT y al personal técnico del laboratorio de corrosión y protección (Jair Lugo Cuevas y Gregorio Vazquez). 


\section{Influencia de Factores Ambientales y Electroquímicos en la Durabilidad del Hormigón}

\section{Resumen}

Este trabajo forma parte del proyecto DURACON, en el cual se caracteriza la durabilidad de concretos expuestos en condiciones ambientales reinantes en Iberoamérica, basándose en la exposición de probetas armadas en al menos dos atmósferas distintas, una marina y una urbana, en cada país iberoamericano participante. Los resultados obtenidos de exponer probetas de concreto armadas a condiciones ambientales reinantes en la $\mathrm{Cd}$. de Chihuahua, México, durante los primeros 15 meses de exposición se discutirán en este trabajo. Se diseñaron dos tipos de hormigones, uno con una relación a/c (agua/cemento) $=0.65$ y otra con $\mathrm{a} / \mathrm{c}=0.45$ $\mathrm{y}$, tres espesores de recubrimiento $(1.5,2.0$ y $3.0 \mathrm{~cm})$. Los ensayos estuvieron centrados principalmente en la medida de parámetros electroquímicos que permitieron evaluar la cinética de corrosión de las armaduras, mediante pruebas de potenciales de corrosión e implementando la técnica de resistencia a la polarización para determinar la velocidad de corrosión. Toda esta información permitirá, con el uso de modelos específicos, diseñar obras de hormigón armado durables y reparar adecuadamente las ya existentes, lo que repercutirá en una disminución de las pérdidas económicas que actualmente está causando el fenómeno de la corrosión en nuestro país.

Palabras clave: corrosión, durabilidad, hormigón armado.

\section{Referencias}

1. C. Andrade, Rev. Iberoamericana de Corrosión y Protección XV (4) (1984).

2. C. Andrade, Cement and Concrete Research 23 (1993) 724-742.

3. C. Andrade, M.A. Sanjuán, A. Escudero, O. Río, Cement and Concrete Research 24 (1994) 1214-1228.

4. C. Andrade, "Manual de inspección de obras dañadas por corrosión de armaduras", Sept. 1988, CSIC, Madrid, España.

5. D.A. Lewism, W.J. Copesnhagen, Industrial Chemist. 11 (1957).

6. American Society for Testing and Materials, (1991), ASTM C 876-91: Standard Test Method for Half-Cell Potentials of Uncoated Reinforcing Steel in Concrete. ASTM, Philadelpia, USA.

7. Oladis Trocónis de Rincón y Miembros de la Red DURAR. "Manual de Inspección, Evaluación y Diagnóstico de Corrosión en Estructuras de Hormigón Armado”. CYTED. Maracaibo, Venezuela. 1997 (1era. Ed.). 